\title{
Epistaxis as the First Manifestation of Silent Renal Cell Carcinoma: A Case Report with Relevant Literature Review
}

\author{
Seung Min Lee, ${ }^{1}$ You Me Kim, and Bong Man Kim ${ }^{1, *}$ \\ ${ }^{1}$ Department of Radiology, Dankook University Hospital, Choenan, South Korea \\ ${ }^{*}$ Corresponding author: Bong Man Kim, Department of Radiology, Dankook University Hospital, Choenan, South Korea. Tel: +82-1056908768, Fax: +82-415509674, \\ E-mail: handuri78@naver.com
}

Received 2015 July 9; Accepted 2015 August 21.

\begin{abstract}
The paranasal sinuses are known to be a rare location for metastasis. Renal cell carcinoma (RCC) is the most frequent primary tumor to metastasize to the sinonasal region, followed by lung and breast cancer. In particular, clear cell type RCC, which represents approximately $85 \%$ of RCCs, is characterized by early metastasis, and it sometimes spreads to unusual sites $(1,2)$. Metastatic tumors in the paranasal sinuses are distributed in the maxillary, sphenoid, ethmoid, and frontal sinuses, in order of decreasing frequency. Symptoms are usually nonspecific, but epistaxis is the most common sign, due to the hypervascularity of the primary tumor. The prognosis is uncertain, but the 5-year survival rate fluctuates between $15 \%$ and $30 \%$. The purpose of this case report is to document a rare case of silent RCC that first presented as epistaxis due to nasal cavity and ethmoid sinus metastasis.
\end{abstract}

Keywords: Metastatic Renal Cell Carcinoma, Sinonasal Metastasis, Epistaxis

\section{Introduction}

Metastasis of renal cell carcinoma (RCC) to the nasal cavity is an extremely rare occurrence, although RCC is the most common infraclavicular primary tumor that metastasizes to the nasal cavity and paranasal sinuses (3). Up to the present, 105 cases of maxillary metastases and 21 cases of ethmoid metastases from renal carcinomas have been reported (4). Nasal metastasis as the initial presentation of RCC is even rarer. We report a case of silent clear cell RCC that presented with epistaxis due to nasal cavity metastasis, followed by a brief review of the relevant literature.

\section{Case Presentation}

A 62-year-old man presented to our ENT department with a 3-day history of massive epistaxis of about 300 $400 \mathrm{ml}$. He had a past medical history of hypertension and diabetes mellitus, without any abnormal previous nasal or urinary symptoms. The initial laboratory findings showed a low hemoglobin level of $8.0 \mathrm{~g} / \mathrm{dL}$ (normal range $13-17 \mathrm{~g} / \mathrm{dL}$ ) but no evidence of thrombocytopenia or coagulopathy, with a platelet count of $330 \times 10^{3} / \mathrm{ml}$ (normal range $130-400 \times 10^{3} / \mathrm{ml}$ ), a prothrombin time of 11.7 seconds (normal range 9 - 13 seconds), an international normalized ratio of 1.00 (normal range $0.7-1.20$ ), and activated PTT of 28.4 seconds (normal range 2 - 37 seconds).

On nasal endoscopy, a very hyperemic polypoid mass in the right nasal cavity was noted (Figure 1A). Computed tomography (CT) of the paranasal sinuses revealed an approximately $2.2 \mathrm{~cm}$, oval-shaped, soft-tissue-density mass on the pre-contrast images, with avid enhancement in the right ethmoid sinus and nasal cavity (Figure 1B). On magnetic resonance imaging (MRI), the lesion appeared hyperintense on T2 weighted imaging (T2WI) and isointense on T1WI, with strong enhancement (Figure 1C). The clinically suspected diagnosis was a hypervascular sinonasal tumor, such as a hemangiopericytoma, hemangioma, or angiofibroma. Endoscopic removal of the right sinonasal mass was performed, and the pathology report suggested metastatic clear cell carcinoma of renal origin. Workup of the primary tumor was initiated in order to screen the abdomen and the pelvic cavity. On contrast-enhanced 3D CT imaging of the kidneys, a heterogeneously enhancing lobulated mass measuring $9.7 \times 8.5 \mathrm{~cm}$ was found in the upper polar region of the left kidney (Figure 1D). The low-density central portion of the mass represented necrosis, and prominent collateral vessels were seen around the mass. The anterolaterally displaced left proximal ureter was completely engulfed by tumor, and increased perirenal soft-tissue-density strands suggested perirenal fat invasion. The patient underwent left radical nephrectomy, and the mass was pathologically confirmed as clear cell type RCC. He remained asymptomatic five months after nephrectomy, and is undergoing further follow-up.

Copyright (C 2016, Tehran University of Medical Sciences and Iranian Society of Radiology. This is an open-access article distributed under the terms of the Creative Commons Attribution-NonCommercial 4 .0 International License (http://creativecommons.org/licenses/by-nc/4.0/) which permits copy and redistribute the material just in noncommercial usages, provided the original work is properly cited. 

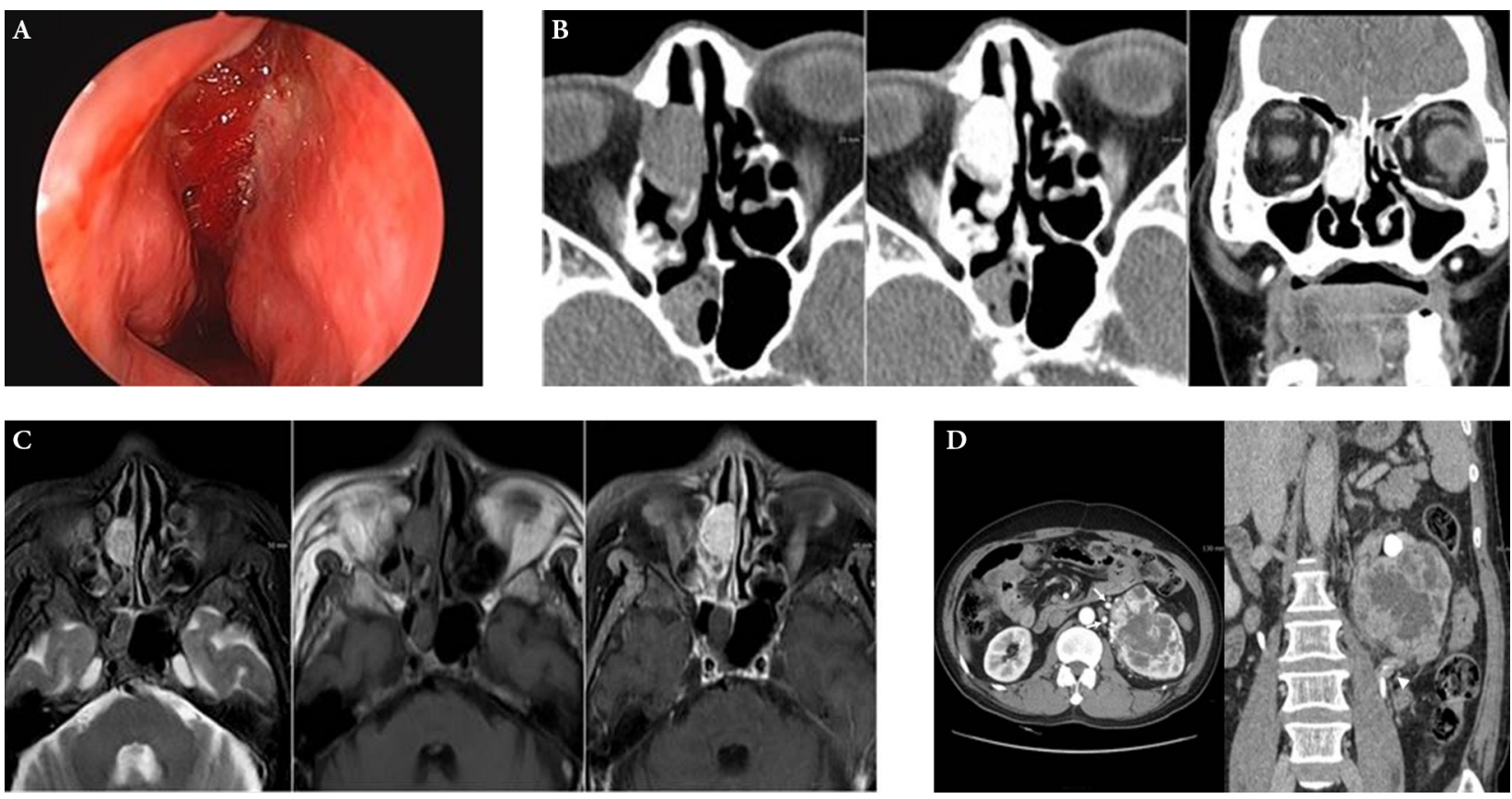

Figure 1. Sixty two-year-old man with nasal metastasis from silent renal cell carcinoma. A, On nasal endoscopy, a hyperemic and hemorrhagic nasal lesion occupying the right middle meatus was noted; B, Axial CT scan showed an oval-shaped, soft-tissue-density mass in the right ethmoid sinus, extending into the nasal cavity with avid enhancement; C, On MRI, a $2.2 \mathrm{~cm}$, oval-shaped, right sinonasal mass appeared hyperintense on T2WI and isointense on T1WI, with strong enhancement; D, On contrast-enhanced 3D CT images of the kidneys, a heterogeneously enhancing lobulated mass measuring $9.7 \times 8.5 \mathrm{~cm}$ was found in the upper polar region of the left kidney. Round high-densities represent prominent vascular collaterals. The tumor mass engulfed the left proximal ureter with irregular luminal narrowing (arrowhead).

\section{Discussion}

Many ear, nose, and throat (ENT) problems are observed in general practice, and epistaxis is one of the common chief complaints of visiting patients. When an underlying hypervascular mass is detected in the nasal cavity or paranasal sinuses, a primary sinonasal tumor is first suspected, such as angiofibroma, hemangiopericytoma, hemangioma, or sinonasal glomus tumors (5). However, a small minority of patients who present with epistaxis show a metastatic secondary sinonasal mass. Therefore, vascular malignant lesions, such as adenocarcinomas, melanomas, and other metastatic tumors from primary sites arising below the level of the clavicle should be considered. Lung, breast, and renal cell carcinomas are common neoplasms that metastasize to the head and neck region; most of these metastases occur in the thyroid gland. However, RCC is the most frequent infraclavicular tumor to metastasize to the nasal cavity and paranasal sinuses (6). Therefore, high suspicion and detection of a secondary nasal mass as the cause of epistaxis, and identification of the primary site of origin, are important in cases of unusual or uncontrolled epistaxis, and RCC metastasis should be included in the differential diagnosis of nasal bleeding lesions.

RCC encompasses a histologically diverse group of solid renal tumors. The most common histologic subtype is clear cell RCC (85\%) (7). Clear cell RCC is known to be associ- ated with loss of function of the von Hippel-Lindau gene, which upregulates hypoxia-induced factor (HIF), finally increasing the function of vascular endothelial growth factor (VEGF). This sequence of events eventually increases the angiogenesis and vascularity of clear cell RCCs and related metastases. Therefore, sinonasal metastases of RCC are characteristically prone to severe nasal bleeding.

It has been accepted that RCC tumor cells spread to the sinonasal region via two potential hematogenous routes: one bypassing pulmonary capillary filtration and the other leading directly to the head and neck region via extensive anastomosis between the avalvular vertebral venous plexus and the intracranial venous plexus (8). The tumor cells spread on the first route via the classical pathway through the inferior vena cava, lungs, heart, and maxillary artery; in these cases, concurrent lung or brain metastases may be present. The second route is via the vertebral plexus, in which tumor cells do not enter the inferior vena cava but travel through the vertebral venous plexus, the intracranial venous plexus, and the cavernous venous plexus via venous anastomoses, to reach the nasal and paranasal sinuses. In these cases, the sinonasal region may be the only site of metastasis.

Many RCCs grow slowly, and only manifest after a considerable tumor size is reached, usually with the classical presentation of flank pain, palpable flank mass, or gross 
hematuria. Therefore, many asymptomatic silent RCCs are difficult to suspect and diagnose. Since symptoms of metastatic tumors often precede those of the primary tumor in renal cancer, when a patient presents with a mass in the sinonasal region, the possibility of a metastatic secondary mass, especially of renal origin, should be considered even in the absence of a past history of renal cancer. The prognosis of patients with metastatic RCC is poor, with a median survival of $7-11$ months. In addition, metastatic RCC is resistant to chemotherapy and radiotherapy, although a variable response has been reported (9). Many metastatic tumors of renal origin develop in multiples, such as in the lung or liver, but most metastatic tumors in the sinonasal region are single. Therefore, a patient with a single, resectable, metastatic RCC in the sinonasal cavity may benefit from surgical intervention, and should be treated aggressively with metastasectomy and primary tumor resection. These patients may expect extended survival before further disease progression.

In conclusion, the case described here is unusual because the presence of a primary tumor was unclear, and because of the unusual metastatic site. Early diagnosis and surgical removal of both primary and metastatic tumors is important in order to prolong the patient's life. When a patient presents with a mass in the nasal or paranasal sinus region, the possibility of metastatic renal cell carcinoma should be considered.

\section{Footnotes}

Authors' Contribution:Study concept and design: You me Kim, Seung min Lee; analysis and interpretation of data: Seung min Lee; drafting of the manuscript: Bong man Kim, Seung-min Lee; critical revision of the manuscript for important intellectual content: You me Kim.

Financial Disclosure:The authors have no financial disclosures to declare.

Funding/Support:This study was supported in part by the genitourinary section of the diagnostic radiology in Dankook university hospital.

\section{References}

1. Roder D, de Silva P, Zorbas H, Kollias J, Malycha P, Pyke C, et al. Survival from synchronous bilateral breast cancer: the experience of surgeons participating in the breast audit of the Society of Breast Surgeons of Australia and New Zealand. Asian Pac J Cancer Prev. 2012;13(4):1413-8. [PubMed: 22799341]

2. Bianchi M, Sun M, Jeldres C, Shariat SF, Trinh QD, Briganti A, et al. Distribution of metastatic sites in renal cell carcinoma: a population-based analysis. Ann Oncol. 2012;23(4):973-80. doi: 10.1093/ annonc/mdr362. [PubMed: 21890909]

3. Prescher A, Brors D. [Metastases to the paranasal sinuses: case report and review of the literature]. Laryngorhinootologie. 2001;80(10):583-94. doi: 10.1055/s-2001-17835. [PubMed: 11602931]

4. Maheshwari GK, Baboo HA, Patel MH, Usha G. Metastatic renal cell carcinoma involving ethmoid sinus at presentation. J Postgrad Med. 2003;49(1):96-7. [PubMed: 12865584]

5. Razek AA, Huang BY. Soft tissue tumors of the head and neck: imaging-based review of the WHO classification. Radiographics. 2011;31(7):1923-54. doi: 10.1148/rg.317115095. [PubMed: 22084180]

6. Leon B, John WE, Peter R, David S. Word Health Organization Classification of Tumours: Pathology and genetics of head and neck tumors. France, Lyon: International Agency for Research on Cancer (IARC); 2005.

7. Karumanchi SA, Merchan J, Sukhatme VP. Renal cancer: molecular mechanisms and newer therapeutic options. Curr Opin Nephrol Hypertens. 2002;11(1):37-42. [PubMed: 11753085]

8. Boles R, Cerny J. Head and neck metastases from renal carcinomas. Mich Med.1971;70(16):616-8. [PubMed:5571989]

9. Ather MH, Masood N, Siddiqui T. Current management of advanced and metastatic renal cell carcinoma. Urol J. 2010;7(1):1-9. [PubMed: 20209445] 